\title{
Remote Method Invocation
}

\section{Goal of RMI}

Implement distributed objects.

Have a program running on one machine invoke a method belonging to an object whose execution is performed on another machine.

\section{Remote Object Technologies}

\section{CORBA}

- Common Object Request Broker Architecture

- Designed for interoperability between different languages as well as different machines.

\section{DCOM or COM}

- Distributed Component Object Model

- Interoperability between different machines and languages as long as they are Wintel.

\section{RMI}

- Java only.

- Interoperability for any machines that are running the Java virtual machine.

\section{Remote versus Local Objects}

Although we would like remote objects to behave exactly the same as local object, that is impossible for several reasons:

- Networks can be unreliable.

- References on one machine (memory addresses) have no meaning on another machine. 


\section{Consequences}

- Special exceptions will indicate network failures.

- Objects passed as parameters and returned as results are passed by copy mode (pass by value).

\section{Reasons for Using RMI}

- Some operations can be executed significantly faster on the remote system than the local client computer.

- Specialized data or operations are available on the remote system that cannot be replicated easily on the local system, for example, database access.

- Simpler than programming our own TCP sockets and protocols.

\section{Overview of an RMI System}

Local Code
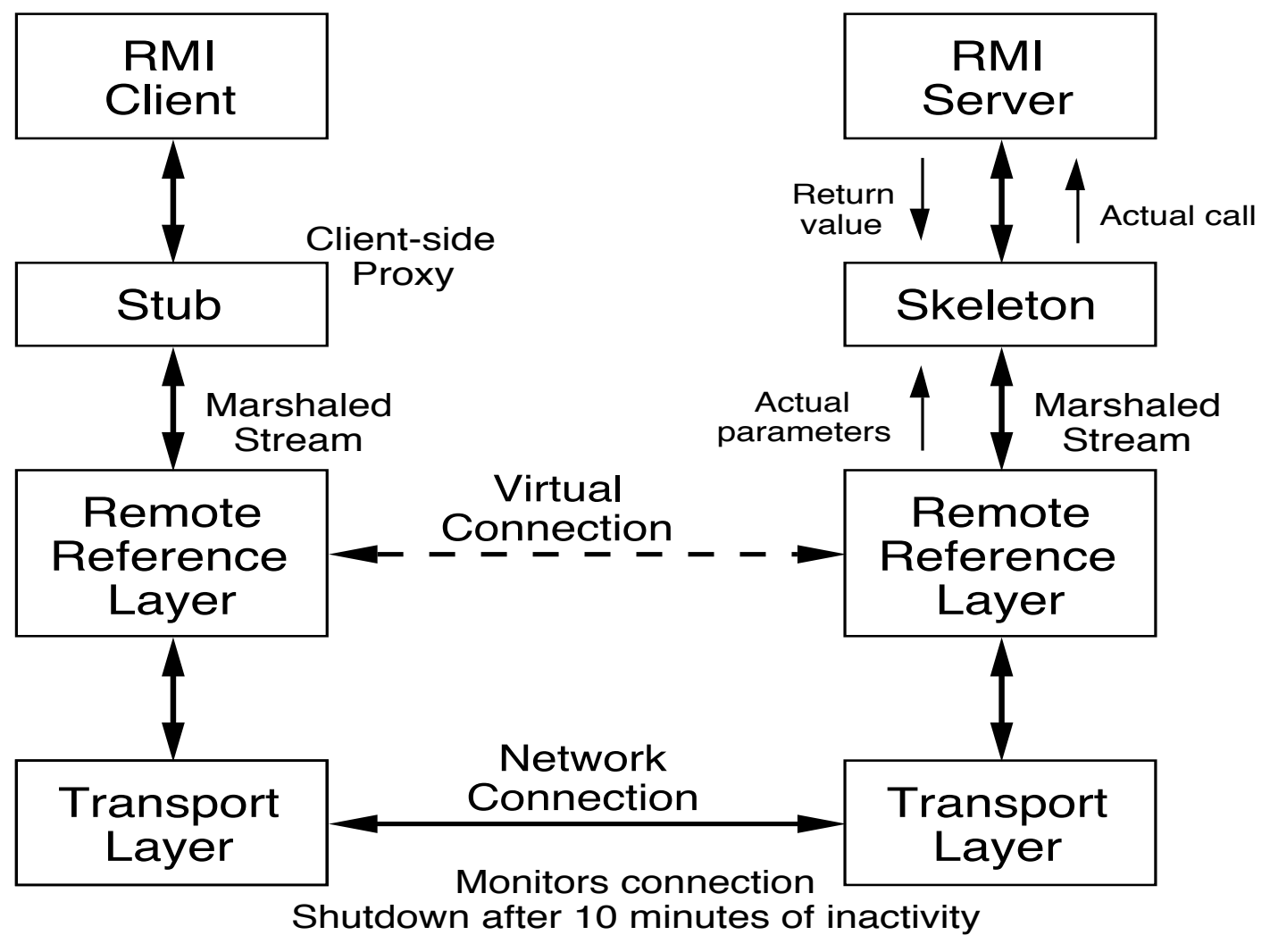

Remote Object

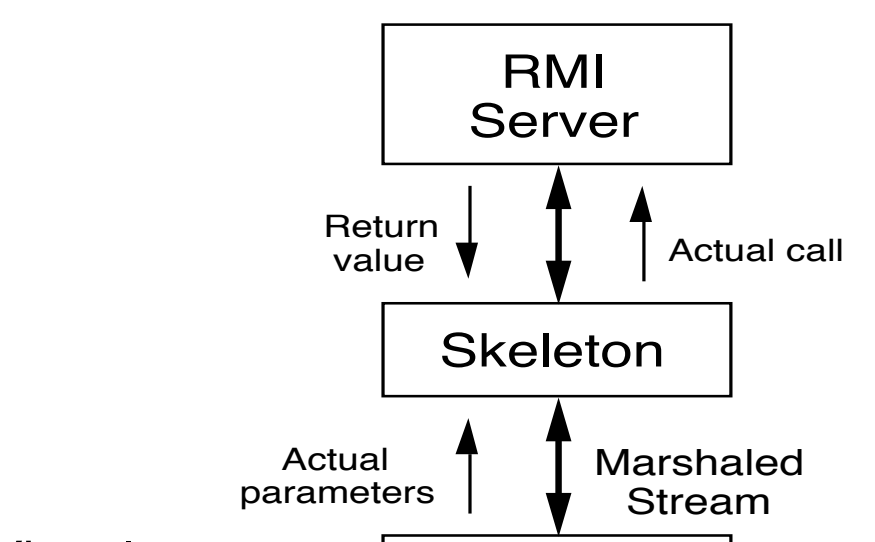

Virtual 


\section{Recipe for RMI}

1. Create and compile an interface that specifies the methods that will have remote access.

- Must be public

- Must extend the interface java.rmi.Remote

- Each remote method must have a throws java.rmi.RemoteException clause

- Interface must reside on both the server and the client side

- Any method parameters or return value of a reference type must implement Serializable.

2. Write and compile a class that implements the remote interface in 1.

- Must extend java.rmi.server.UnicastRemoteObject

- Must implement the remote interface in 1

- Its constructors must be defined explicitly since they each may throw java.rmi.RemoteException

- Resides on the server side only

3. Create the stub class using the rmic tool.

\section{$\%$ rmic -v1.2 ImplementationClass}

- Leave the stub on the server side

- Put a copy of the stub class on the client side

4. Write and compile a server class that

- Installs a security manager using 
System.setSecurityManager(

new java.rmi.RMISecurityManager());

This step may be optional.

- Instantiates an object of the implementation class in 2; this is the remote object

- Binds the remote object implObj to a unique identifier (a String) for the rmi registry java.rmi.Naming.rebind("uniqueID", implObj);

Note: The implementation class and the server class can be combined into one class, particularly if there is no need for the server class to extend another class.

5. Write and compile a client class that

- Installs a security manager as in 4 (this step may be optional also).

- Requests an object from the remote server using its hostname and the unique identifier of the object, and then casts that object to the interface type from 1.

InterfaceType it =

(InterfaceType)java.rmi.Naming.lookup(

"rmi://r-Inx233.cs.uiowa.edu:1099/uniquelD");

1099 is the default port and if used, its specification here can be omitted.

- If the client is running on the same machine as the remote object (the server), use 
InterfaceType it =

(InterfaceType)java.rmi.Naming.lookup(

"rmi://localhost/uniqueID");

or

InterfaceType it $=$

(InterfaceType)java.rmi.Naming.lookup("rmi:///uniqueID");

- Now methods on the remote object can be called using the interface class object it when the rmi system is up and running.

6. Start the bootstrap rmi registry in the background on the server side.

$\%$ rmiregistry \&

$\%$ rmiregistry $1492 \&$
// assumes port 1099

// specifies a port

7. Execute the server class on the machine that the client named, which was r-Inx233.

\section{$\%$ java ServerClass}

Note: The rmi registry and the server must run on the same machine and in the same directory.

8. Execute the client class on another machine $\%$ java ClientClass

Note: Any objects that will be passed as parameters to the remote object method or returned as its result must be Serializable. 


\section{Class Naming Convention}

Employing a uniform pattern of names can help simplify the use of RMI:

Interface:

Name

File

Implementation:

TryRmi

TryRmi.java

Server

TryRmilmpl

TryRmilmpl.java

Client

TryRmiServer

TryRmiClient
TryRmiServer.java

TryRmiClient.java

\section{Server Side Classes}

TryRmi.class

TryRmilmpl.class

TryRmiServer.class

TryRmilmpl_Stub.class

the stub

\section{Client Side Classes}

TryRmi.class

TryRmiClient.class

TryRmilmpl_Stub.class

the stub

\section{Example}

A server receives a String message from a client and returns a String containing the message and an indication of the time elapsed since the last message it handled.

The client provides a window in which a user can type a message to send to the server and a text area where the Strings returned from the server are displayed. 


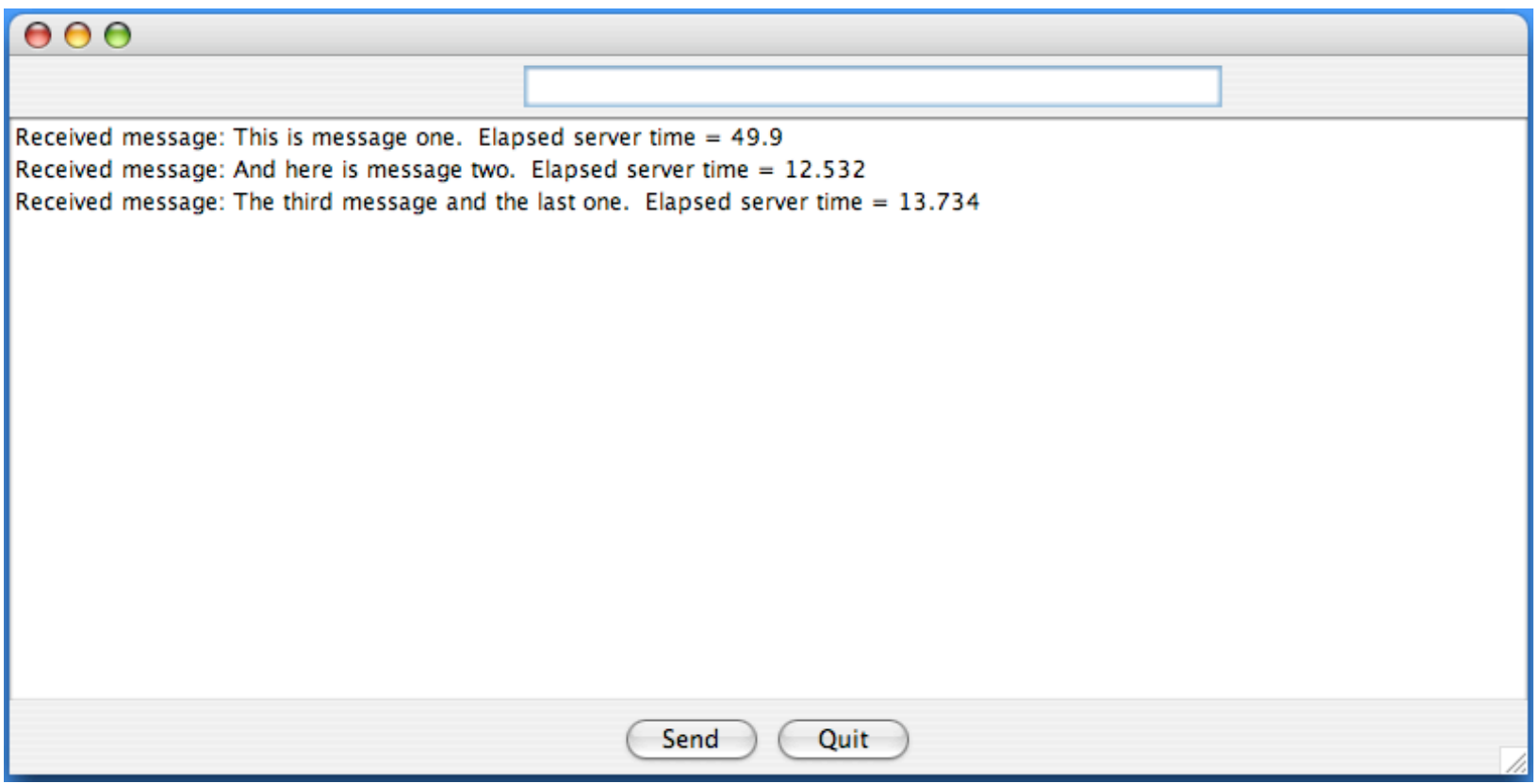

\section{The Code}

import java.rmi. ;

// TryRmi.java

public interface TryRmi extends Remote

\{

String send(String s) throws RemoteException;

\}

$/ /$

import java.rmi. *; $\quad$ // TryRmilmpl.java

import java.rmi.server.UnicastRemoteObject;

public class TryRmilmpl extends UnicastRemoteObject implements TryRmi

\{

private TryRmiServer trs;

private long startTime; 
TryRmilmpl(TryRmiServer $r$ ) throws RemoteException \{

$\operatorname{trs}=r$;

startTime $=$ System.currentTimeMillis();

\}

public String send(String message)

throws RemoteException

\{

trs.report(message);

long now = System.currentTimeMillis();

double time $=($ now - startTime $) / 1000.0$;

startTime = now;

return ("Received message: " + message

+ " Elapsed server time = " + time);

\}

\}

$/ 1-$

import java.rmi. ; // TryRmiServer.java

import java.rmi.server.*;

import java.net.InetAddress;

import java.rmi.registry.*; // Registry, LocateRegistry

public class TryRmiServer

\{

TryRmiServer()

\{

System.setSecurityManager(

new RMISecurityManager()); 


\section{try}

\{ TryRmi talk = new TryRmilmpl(this);

Naming.rebind("TryRmi", talk);

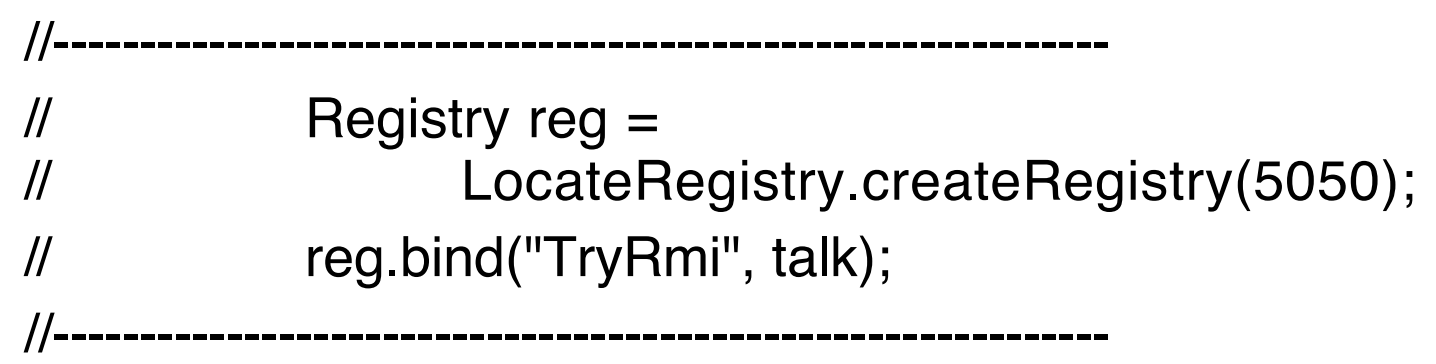

System.out.printIn("Server ready on "

+ InetAddress.getLocalHost().getHostName());

System.out.printIn("Stop server using control-c");

\}

catch (Exception e)

\}

$\{$ System.out.println(e); \}

void report(String text)

\{

System.out.println("Message from client: " + text);\}

public static void main(String [] args)

\{

new TryRmiServer();

\}

\}

$/ 1-$ 
import java.rmi.*;

import java.awt.*;

// TryRmiClient.java

import java.awt.event. ;

import javax.swing. *;

public class TryRmiClient extends JFrame

implements ActionListener

\{

private DefaultListModel model;

private JButton send, quit;

private JTextField sendText;

private TryRmi tryRmi;

private String url ="rmi://r-Inx233.cs.uiowa.edu:5050/TryRmi";

TryRmiClient()

\{

System.setSecurityManager(

new RMISecurityManager());

Container $\mathrm{cp}=$ getContentPane();

JPanel $p=$ new JPanel();

p.add(new JLabel("Enter message: "));

p.add(sendText $=$ new JTextField(30));

cp.add(p, "North");

model $=$ new DefaultListModel();

JList list $=$ new JList $($ model);

JScrollPane jsp = new JScrollPane(list);

cp.add(jsp, " Center ");

$p=$ new JPanel(); 
p.add(send = new JButton("Send"));

send.addActionListener(this);

p.add(quit = new JButton("Quit"));

quit.addActionListener(this);

cp.add(p, "South");

setBounds(100, 100, 800, 400);

setVisible(true);

try

$\{\quad$ tryRmi $=($ TryRmi)Naming.lookup(url);

\}

catch (Exception e)

\{ System.out.println("Naming Exception"+e); \}

\}

public void actionPerformed(ActionEvent e)

\{

if (e.getSource() == quit) System.exit(0);

if (e.getSource ()$==$ send) sendClicked();

\}

private void sendClicked()

\{

String message = "'";

String text $=$ sendText.getText();

sendText.setText("'");

sendText.requestFocus();

try

$\{$ message $=$ tryRmi.send(text); $\}$

catch (Exception e)

\{ System.out.println(e); \} 


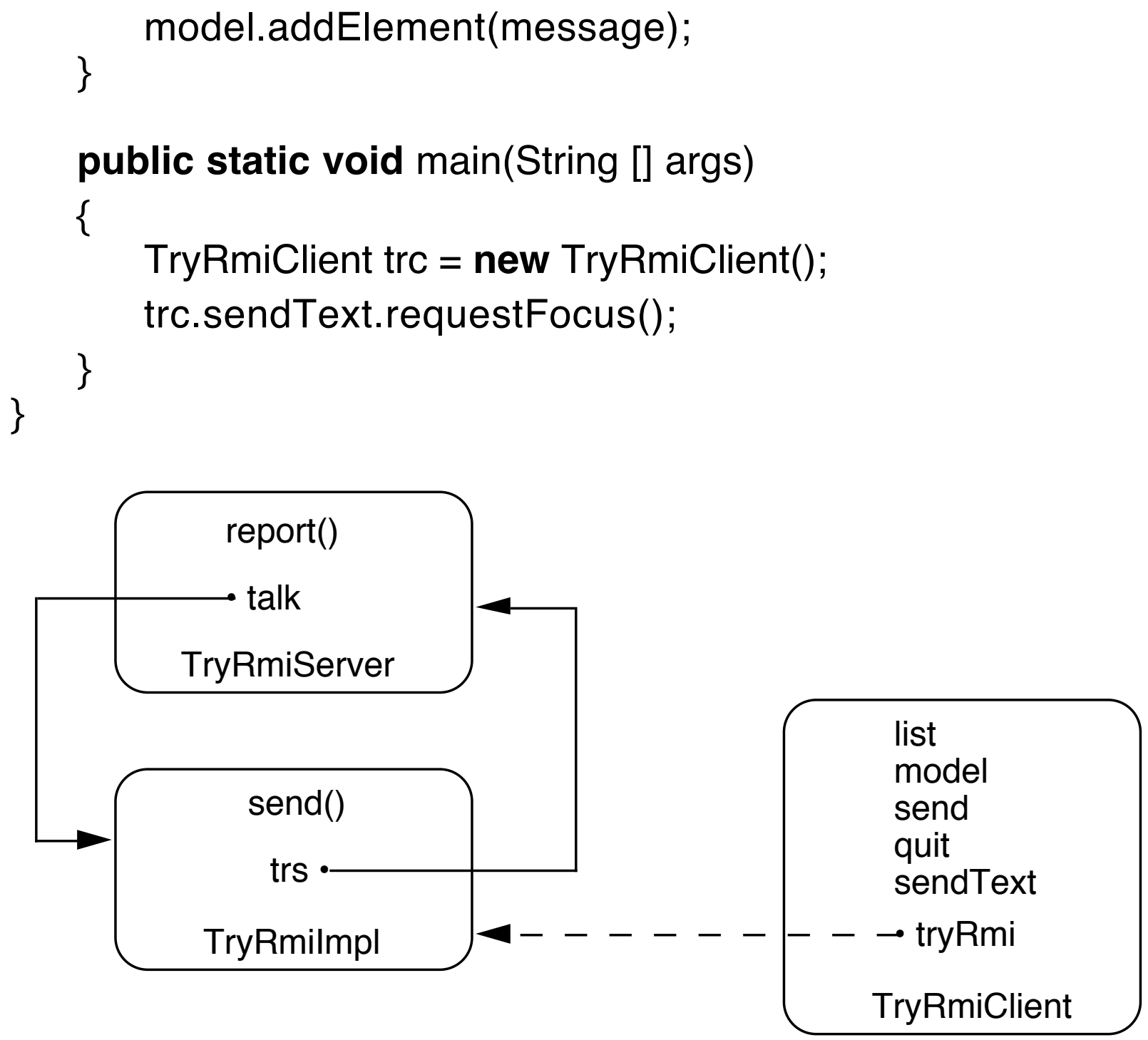

Running TryRmi

$\%$ javac TryRmi.java

$\%$ javac TryRmilmpl.java

$\%$ javac TryRmiServer.java

$\%$ javac TryRmiClient.java

\% rmic -v1.2 TryRmilmpl

Put server classes on server machine, whose name ( $r$-Inx233.cs.uiowa.edu) is hard-coded into the client. 
Put client classes on client machine.

$\%$ rmiregistry $\mathbf{5 0 5 0} \&$ // on r-Inx233.cs.uiowa.edu

$\%$ java TryRmiServer // on r-Inx233.cs.uiowa.edu

$\%$ java TryRmiClient // on any machine

\section{Alternative to rmiregistry (in TryRmiServer)}

import java.rmi.registry. ;

/I-

Registry reg = LocateRegistry.createRegistry(5050);

reg.bind("TryRmi", talk);

$/ /$

\section{Parameter Passing}

Compare passing an object to an instance method for a local object with passing it to a remote object.

The object to be passed will implement an interface so that the example can be developed in a remote context.

The methods in the interface all throw RemoteException eventually for the same reason.

public interface NumsInterface

\{

String $\mathrm{mkStr}()$;

int getM();

int get $\mathrm{N}()$;

void setM(int $k$ );

void setN(int $k)$;

\}

// NumsInterface.java 
The class that implements NumsInterface will provide the objects that are passed as parameters.

// Nums.java

public class Nums implements NumsInterface

\{

private int $m, n$;

Nums(int k1, int k2)

$\{\mathrm{m}=\mathrm{k} 1 ; \mathrm{n}=\mathrm{k} 2 ;$ \}

public String $\mathrm{mkStr}()$

\{

return "Nums[m = " + m + ", $n=$ " + n + "]";

\}

public int getM()

\{ return $\mathrm{m} ;$ \}

public int getN()

\{ return $\mathrm{n} ;$ \}

public void setM(int $k$ )

$\{\mathrm{m}=\mathrm{k} ;$ \}

public void setN(int $k$ )

$\{\mathrm{n}=\mathrm{k} ;\}$

\}

\section{Calling a Method on a Local Object}

import java.io. *;

// LocalTest.java

public class LocalTest

\{ 
public static void main(String [] args) throws Exception \{

Nums nums = new $\operatorname{Nums}(5,13)$;

LocalTest It $=$ new LocalTest();

System.out.println("nums = " + nums.mkStr());

It.alter(nums); // pass object to local object

System.out.printIn("--------------------------");

System.out.println("nums = " + nums.mkStr());

\}

void alter(NumsInterface nm) throws Exception \{

$n m \cdot \operatorname{setM}(2 * n m \cdot \operatorname{get} M())$; $\mathrm{nm} \cdot \operatorname{set} \mathrm{N}(3 * \mathrm{~nm} \cdot \operatorname{get} \mathrm{N}())$;

\}

\}

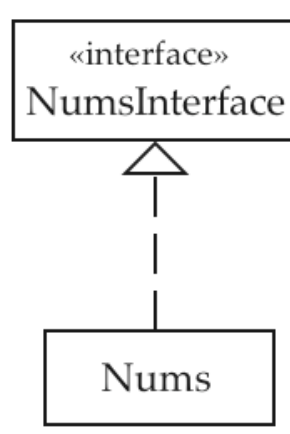

LocalTest alter()

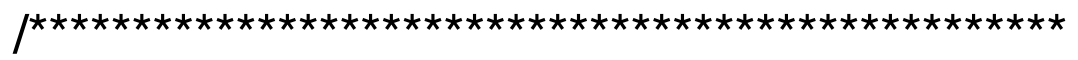

nums $=$ Nums $[m=5, n=13]$

nums $=$ Nums $[\mathrm{m}=10, \mathrm{n}=39]$ 


\section{Calling a Method on a Remote Object}

Now put the alter method into a remote object.

Need an interface for the remote object:

import java.rmi..; // Remo.java

public interface Remo extends Remote

\{

void alter(NumsInterface nm) throws RemoteException;

\}

Then implement the interface with a class that provides the remote object and acts as a server, creating an instance of the remote object and registering it.

import java.rmi.*; // Remolmpl.java

import java.rmi.server.UnicastRemoteObject;

public class Remolmpl extends UnicastRemoteObject

implements Remo

\{

public void alter(NumsInterface $\mathrm{nm}$ )

\{

throws RemoteException

$n m \cdot \operatorname{setM}(2 * n m \cdot g e t M())$;

$\mathrm{nm} \cdot \operatorname{set} \mathrm{N}(3 * \mathrm{~nm} \cdot \operatorname{get} \mathrm{N}())$;

\}

Remolmpl() throws RemoteException

\{

\}

System.out.printIn("Initializing Remote Test");

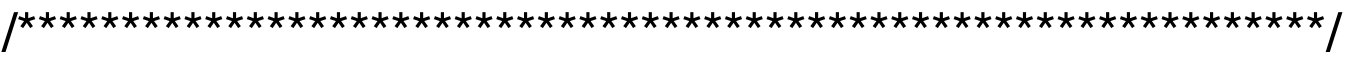


public static void main(String [] args)

\{

// Acts as server also

System.setSecurityManager(new RMISecurityManager());

\section{try}

\{

Remo ri $=$ new Remolmpl();

Naming.rebind("remoteServer", ri);

System.out.printIn("Registered with registry");

\}

catch (RemoteException e)

\{ System.out.println("Remote Error: " + e);

\}

catch (java.net.MalformedURLException e)

\{ System.out.printIn("URL Error: " + e);

\}

\}

We need a client that creates a Nums object and calls alter on the remote object just created, passing the Nums object.

Nums must implement Serializable now.

import java.rmi. ; $\quad$ // Client.java

public class Client

\{

public static void main(String [] args)

\{

System.setSecurityManager(

try

new RMISecurityManager());

\{

NumsInterface nums = new Nums(5, 13);

System.out.println("nums = " + nums.mkStr()); 
Remo remo $=($ Remo $)$ Naming.lookup (

"rmi://localhost/remoteServer");

remo.alter(nums);

System.out.printIn("-------------------------------");

System.out.printIn("nums = " + nums.mkStr());

System.out.println("Done");

\}

catch (NotBoundException e)

\{ System.out.printIn("Server not found"); \}

catch (RemoteException e)

\{ System.out.printIn("Remote error: " + e); \}

catch (java.net.MalformedURLException e)

\{ System.out.printIn("URL error: " + e); \}

\}

\}

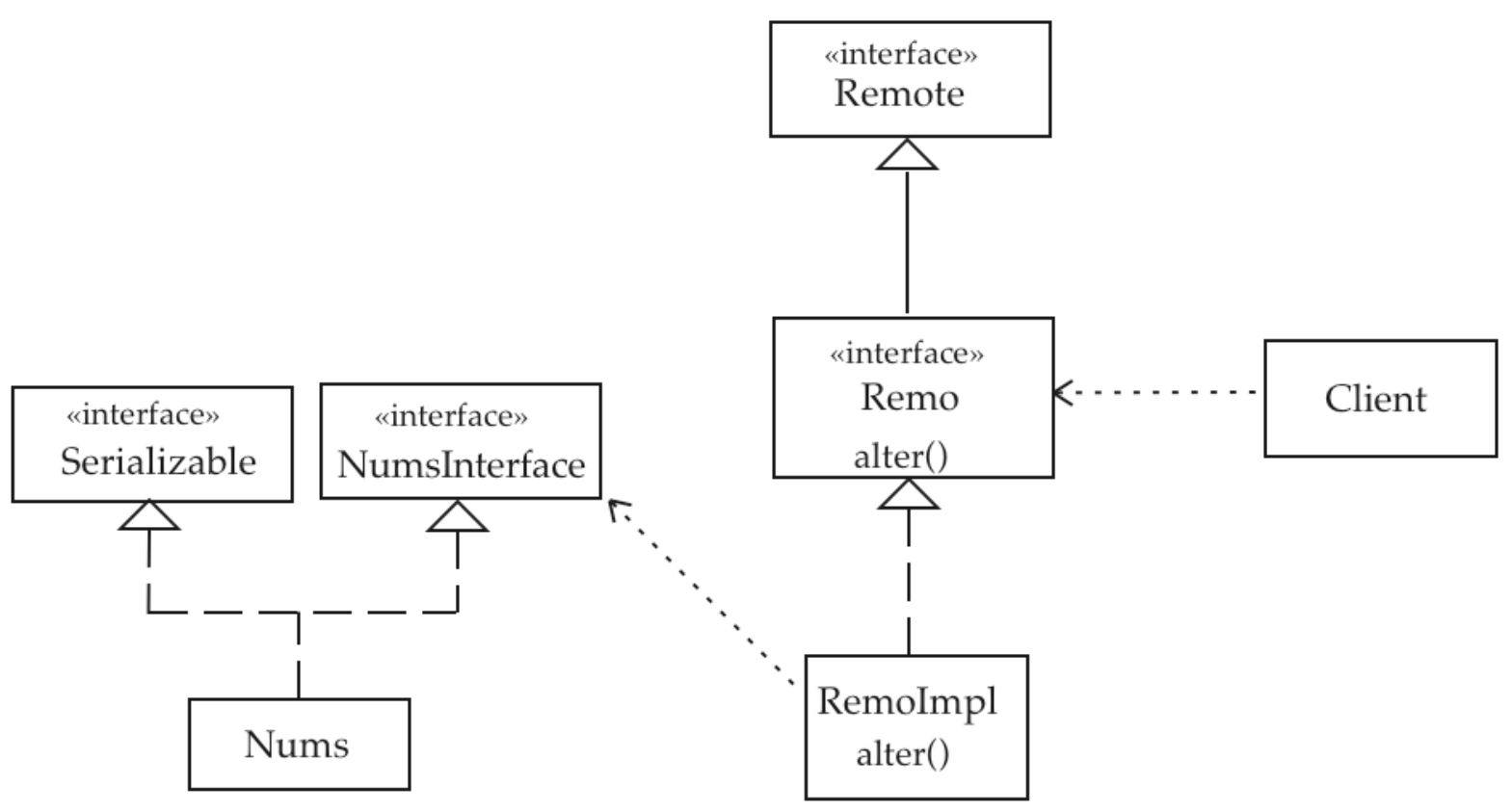


$\%$ rmiregistry \&

[1] 14550

$\%$ java Remolmpl \&

[2] 14558

Initializing Remote Test

Registered with registry

$\%$ java Client

nums $=$ Nums $[m=5, n=13]$

nums $=$ Nums $[m=5, n=13]$

Done

\section{Nums as a Remote Object}

Make NumsInterface into a remote interface called RNumsInterface.

import java.rmi.*; // RNumsInterface.java

public interface RNumsInterface extends Remote \{

String mkStr() throws RemoteException; int getM() throws RemoteException; int getN() throws RemoteException; void setM(int k) throws RemoteException; void setN(int k) throws RemoteException; \}

Change Nums into RNums, a subclass of UnicastRemoteObject. 
import java.rmi.*;

// RNums.java

import java.io.*;

import java.rmi.server.UnicastRemoteObject;

public class RNums extends UnicastRemoteObject

implements Serializable, RNumsInterface

\{

private int $m, n$;

RNums(int k1, int k2) throws RemoteException

$\{\mathrm{m}=\mathrm{k} 1 ; \mathrm{n}=\mathrm{k} 2 ;\}$

public String mkStr() throws RemoteException

\{ return "Nums[m = " + m + ", $n="+n+"] " ;$ \}

public int getM() throws RemoteException

\{return $\mathrm{m} ;$ \}

public int getN() throws RemoteException

\{ return $\mathrm{n} ;$ \}

public void setM(int $k$ ) throws RemoteException

$\{\mathrm{m}=\mathrm{k} ;$ \}

public void setN(int k) throws RemoteException

$\{\mathrm{n}=\mathrm{k} ;\}$

\}

Change the type of the parameter of alter to RNumsInterface in Remo and Remolmpl.

Change Client into RClient, which creates an RNums object to pass to alter. 
import java.rmi. *; // RClient.java

public class RClient

\{

public static void main(String [] args)

\{

System.setSecurityManager(new RMISecurityManager());

try

\{

NumsInterface nums = new $\operatorname{RNums}(5,13)$;

System.out.println("nums = " + nums.mkStr());

Remo remo $=($ Remo $)$ Naming.lookup (

"rmi://localhost/remoteServer");

remo.alter(nums);

System.out.println("------------------------");

System.out.println("nums = " + nums.mkStr());

System.out.println("Done");

\}

catch (NotBoundException e)

\{ System.out.println("Server not found"); \}

catch (RemoteException e)

\{ System.out.println("Remote error: " + e); \}

catch (java.net.MalformedURLException e)

\}

\{ System.out.printIn("URL error: " + e); \}

\} 


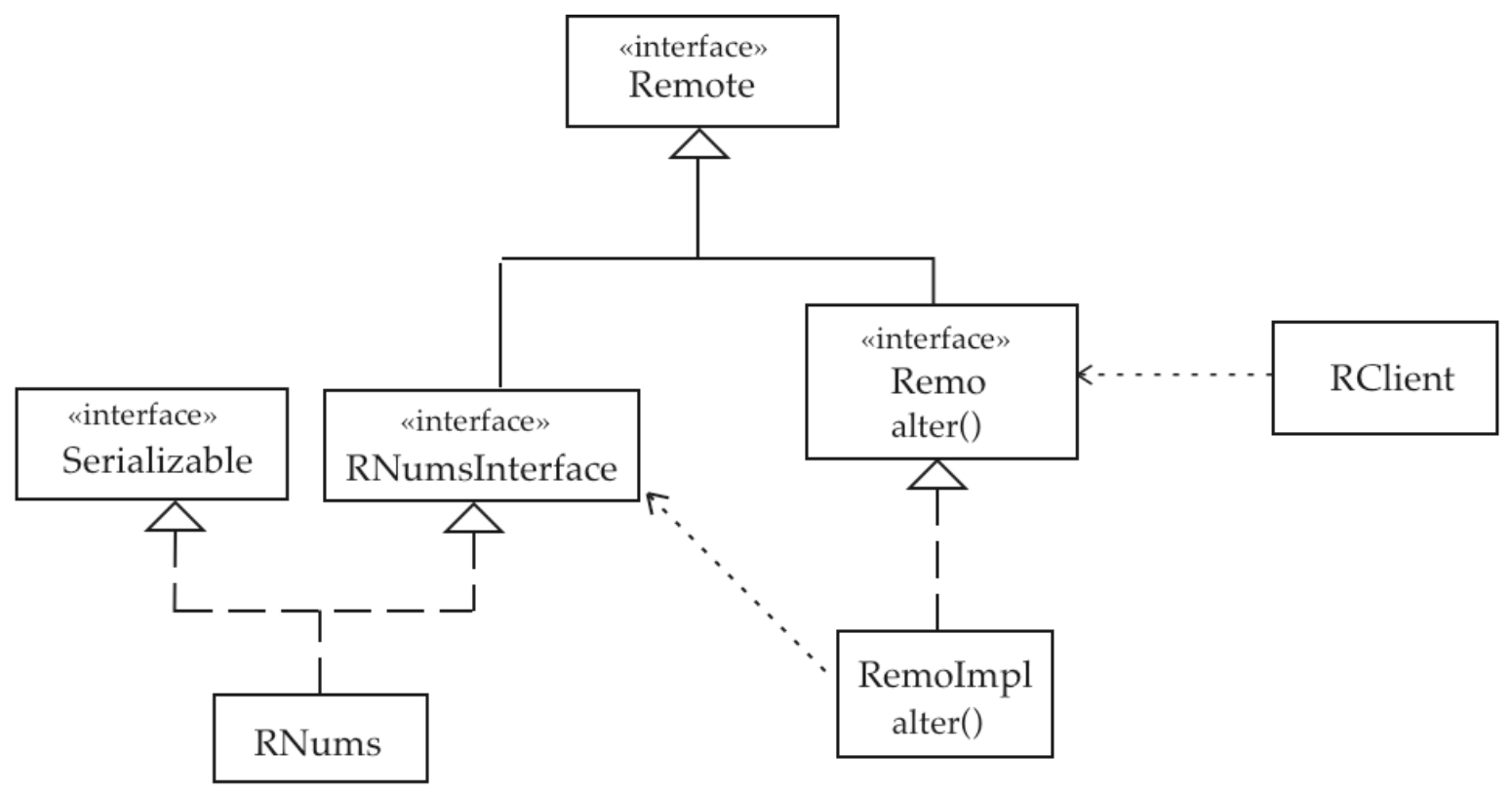

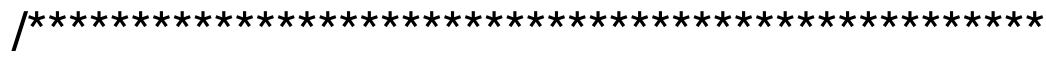

$\%$ rmiregistry \&

[1] 14550

$\%$ java Remolmpl \&

[2] 1558

Initializing Remote Test

Registered with registry

$\%$ java RClient

nums $=$ Nums $[m=5, n=13]$

nums $=\operatorname{Nums}[m=10, n=39]$

Done 
Client side

1. Client

Server side

Remolmpl

implements Remo (Remote)

remo ----------------------> alter(NumsInterface)

2. RClient

Remolmpl

implements Remo (Remote)

remo ----------------------> alter(RNumsInterface)

Calls remo.alter(nums)

where

1. nums $=$ new Nums implements NumsInterface

2. nums $=$ new RNums

implements RNumsInterface (Remote)

\section{client files}

RNumsInterface.class

RNums.class

RClient.class

RNums_Stub.class

Remo.class

Remolmpl_Stub.class

server files

Remo.class

Remolmpl.class

Remolmpl_Stub.class

RNumsInterface.class

RNums_Stub.class 


\section{A Chat System with a Whiteboard}

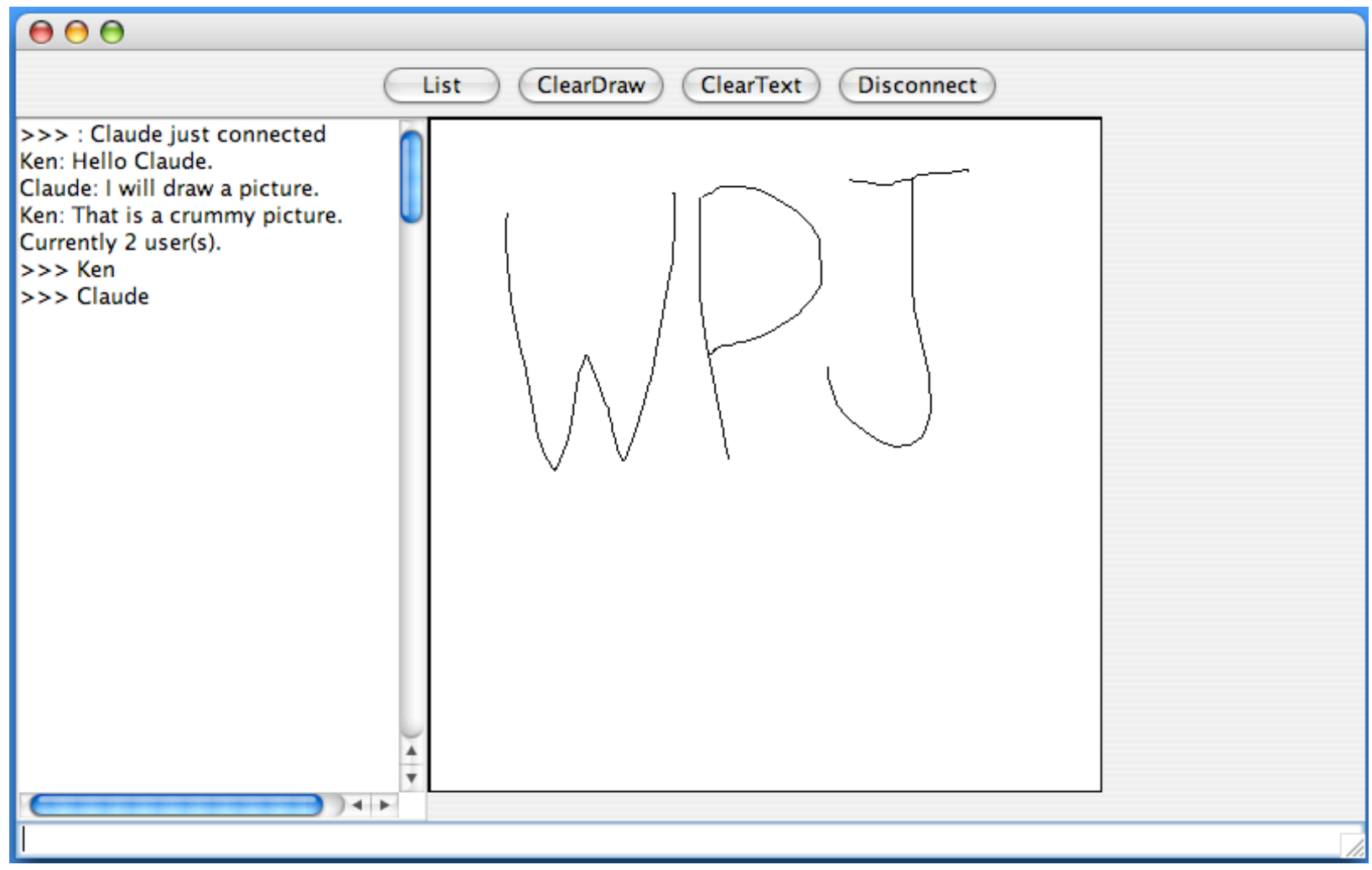

Server running on r-Inx233.cs.uiowa.edu.

One client (Ken) running on cornfed.cs.uiowa.edu (a Mac).

Another client (Claude) running on r-Inx233.cs.uiowa.edu.

Note: I had trouble with getting the JDialog to show on cornfed, so I hard-coded the name of the user on that machine. 


\section{Actions}

A client obtains a reference to a remote object (ChatServer implemented by ChatServerlmpl) that provides three remote methods:

register

postMessage

listChatters

When a client registers with the server, it passes a remote reference to itself (ChatClient implemented by ChatClientImpl) that provides one method: chatNotify.

Note that the remote reference passed to register must be of the interface type, ChatClient, not of the object type ChatClientImpl.

Therefore, the server has a remote reference to each of its clients so that it can notify each, using chatNotify, when a new message has arrived.

The chatNotify method acts as a callback function that the server uses to pass messages to the clients.

Since clients will be notified of messages that are broadcast, they do not have to poll the server but just wait until the server notifies them of a new message.

On the client side, each client has a thread, an instance of ServerTalker, that maintains a queue of messages to be set to the server using postMessage, one of the methods that the server remote object recognizes.

As long as the message queue is not empty, the messages are removed and sent to the server. 
The threads ensure that no client is delayed waiting for a remote method invocation on the server to complete.

Each client can be attentive to user input.

On the server side, the server also has a thread for each client, an instance of ClientTalker, so that a slow client will not delay the system.

Each ClientTalker object maintains a queue of messages to be sent to its client using the callback method chatNotify, the one method that the client remote object recognizes.

When a client calls postMessage, that method calls a boolean method addMessage in each ClientTalker to add the message to its queue.

This method returns false if its client is no longer connected.

The ChatServerlmpl object starts the registry in its main method using

Registry reg = LocateRegistry.createRegistry(5050); before binding itself to the unique identifier "ChatServerImpl".

\section{Message Objects}

- The class Message implements Serializable so that its objects can be sent as parameters from a client to the server and then back to each of the clients.

- Message objects include the name of the client who initiated the message.

- Message objects may encapsulate a String or a Line.

Since ChatClientlmpl extends JFrame, it cannot be a subclass of UnicastRemoteObject. 
Therefore, the remote interface must be exported manually using the command:

UnicastRemoteObject.exportObject(this);

Once a client is registered with the server, it remains idle until either

- A user event (text or a line) causes a message to be sent to the server by ServerTalker or a button is pressed.

or

- Its method chatNotify is called by the server, passing a message back.

When a message comes back by means of chatNotify, either

- Text data is appended to the TextArea

or

- A Line object is added to an off-screen buffer, which is shown when the panel of the whiteboard is redrawn.

When the ServerTalker has emptied its queue of messages, the client becomes idle again.

\section{The Whiteboard}

When the mouse is dragged on the panel that makes up the whiteboard, lines are created and placed in an internal buffer, a List, and then the render method is called.

Those lines will be drawn on an off-screen image buffer and sent as messages to the server when the render method is executed.

The offscreen image is drawn on the panel inside paintComponent, provided the image exists. 


\section{The Code}

import java.rmi.*; // ChatServer.java

public interface ChatServer extends Remote \{

void register(ChatClient c,String name)

throws RemoteException;

void postMessage(Message $\mathrm{m}$ )

throws RemoteException;

String [] listChatters() throws RemoteException;

\}

$/ /$

import java.rmi..; $\quad$ // ChatServerlmpl.java

import java.rmi.server.*;

import java.rmi.registry. ;

import java.util.*;

public class ChatServerlmpl extends UnicastRemoteObject implements ChatServer

\{

private List $<$ ClientTalker $>$ chatters $=$

new ArrayList<ClientTalker $>()$;

ChatServerlmpl() throws RemoteException

\{

System.out.printIn("Initializing Server...");

\}

public static void main(String [] args)

\{

System.setSecurityManager(new RMISecurityManager()); 
try

\{

ChatServer csi $=$ new ChatServerImpl();

Registry reg $=$

LocateRegistry.createRegistry(5050);

reg.rebind("ChatServerImpl", csi);

System.out.printIn("Server Ready");

\}

catch (RemoteException e)

\}

\{ System.out.printIn("ChatServer Error: " + e); \}

public synchronized void register(ChatClient $c$, String $n$ )

\{ throws RemoteException;

chatters.add(new ClientTalker(c, n));

System.out.println( $n+$ " added to chat group.");

\}

// Note remote object parameter of type ChatClient.

public synchronized void postMessage(Message $\mathrm{m}$ )

\{ throws RemoteException;

for (Iterator it=chatters.iterator(); it.hasNext; )

\{

ClientTalker ct $=($ ClientTalker)it.next () ;

boolean alive $=$ ct.addMessage $(\mathrm{m})$;

if (!alive) it.remove();

\}

\}

public String [] listChatters()throws RemoteException; \{

String [] list = new String[chatters.size()]; 
for (int $k=0$; $k<$ list.length; $k++$ )

\{

ClientTalker $\mathrm{ct}=$ chatters.get $(\mathrm{k})$;

list $[\mathrm{k}]=$ ct.getChatterName();

\}

\}

return list;

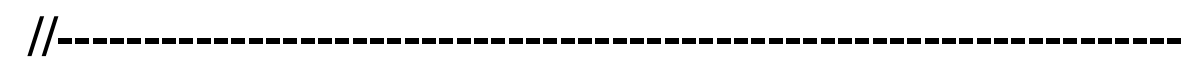

import java.util.*; // ClientTalker.java import java.rmi.*;

public class ClientTalker extends Thread \{

private List $<$ Message $>$ messages $=$

new LinkedList<Message $>()$;

private ChatClient chat;

boolean isActive = true;

private String name;

ClientTalker(ChatClient c, String $\mathrm{n}$ )

$\{$ chat $=\mathrm{C}$;

name $=\mathrm{n}$;

start();

\}

synchronized boolean addMessage(Message e) \{

if (lisActive) return false;

messages.add (e);

notifyAll();

\}

return true; 


\section{public synchronized void run()}

\{

boolean running = true;

while (running)

$\{$ try

$\{$ if (messages.isEmpty ()$)$ wait () ;

chat.chatNotify((Message)messages.get(0));

messages.remove(0);

\}

catch (InterruptedException e)

\{ System.out.printIn("Interrupted: " + e); \}

catch (RemoteException e)

\{

System.out.println("Removing "+ name);

is Active=false;

running = false;

\}

yield();

\}

\}

String getChatterName()

\}

\{ return name; \}

$/ /$

import java.io.*; // Message.java

public class Message implements Serializable \{

private String sender = null;

private String text = null;

private Line line = null; 


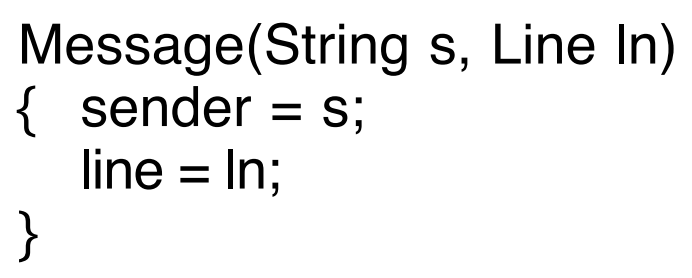


$/ /-$

import java.rmi. *; // ChatClient.java

public interface ChatClient extends Remote

\{

\}

void chatNotify(Message m) throws RemoteException;

$/ 1$

import java.rmi. *; // ChatClientImpl.java

import java.rmi.server. *;

import java.net.*;

import java.awt.*;

import java.awt.event. *;

import java.util.*;

import javax.swing. *;

public class ChatClientImpl extends JFrame implements ChatClient, LineSender, ActionListener

\{

private JTextArea ta; // display chatter

private JTextField tf; // message to send

private ChatServer cs; // reference to server private String name = null; // user's name

private DrawPad dp; // whiteboard private ServerTalker st;

ChatClientImpl() throws RemoteException \{

JButton list, cDraw, cText, dis;

System.out.printIn("Starting up Chat Client");

Container $\mathrm{cp}=$ getContentPane(); 
JPanel $p=$ new JPanel();

p.add(list = new JButton("List")); // list users

list.addActionListener(this);

p.add (cDraw = new JButton("ClearDraw"));

cDraw.addActionListener(this);

p.add(cText = new JButton("ClearText"));

cText.addActionListener(this);

p.add(dis = new JButton("Disconnect"));

dis.addActionListener(this);

cp.add(p, "North");

ta $=$ new JTextArea $(150,20) ; \quad$ // transcript

cp.add(ta, "West");

$\mathrm{dp}=$ new DrawPad(this);

// whiteboard

cp.add(dp, "Center");

tf = new JTextField (40);

// text entry field

tf.addActionListener(this);

\}

cp.add(tf, "South");

void registerChatter()

\{

NameDialog nd $=$ new

NameDialog(new JFrame("Enter Name"),

"Enter your name", false);

name $=$ nd.getName();

nd.setVisible(false); // get rid of NameDialog

nd = null;

String url =

"rmi://r-Inx233.cs.uiowa.edu:5050/ChatServerImpl";

System.setSecurityManager(new RMISecurityManager());

try

\{ 
UnicastRemoteObject.exportObject(this);

// export remote methods

$c s=($ ChatServer $)$ Naming.lookup(url);

st = new ServerTalker(cs, name);

cs.register(this, name);

// remote call

\}

catch (RemoteException e)

\{

System.out.println("Cannot find Registry ");

System.exit(0);

\}

catch (MalformedURLException e)

\{

System.out.printIn("Bad URL: " + url);

System.exit(0);

\}

catch (NotBoundException e)

\{

System.out.println("Service not bound." );

System.exit(0);

\}

tf.requestFocus();

\}

public void actionPerformed(ActionEvent e)

\{

if $($ e.getSource ()$==$ tf)

\{

boolean alive $=$ st.addMessage (

new Message(name, tf.getText().trim()));

if (!alive) ta.append("*** Server Error $\left.{ }^{* \star \star} \backslash n "\right)$;

tf.setText("'");

\}

if (e.getSource() instanceof JButton)

\{ 
String arg $=$ e.getActionCommand () ;

if (arg.equals("ClearText")) ta.setText("');

else if (arg.equals("ClearDraw")) dp.clearScreen();

else if (arg.equals("Disconnect"))

\{

st.addMessage(new Message(">> " + name,

System.exit(0);

"Logged off. Bye"));

\}

else if (arg == "List") getUserList();

\}

\}

tf.requestFocus();

public void sendLine(Line In)

\{

boolean alive $=$ st.addMessage $($ new Message $($ name,$I n))$;

\}

if (!alive) ta.append("*** Server Error ${ }^{* * *}(n ")$;

void getUserList()

\{

String [] users = null;

try

$\{\quad$ users $=$ cs.listChatters () ;

\}

catch (RemoteException e)

\{

System.out.println(e);

users = new String[1];

\}

users $[0]=" * * *$ Error ${ }^{* * * " ;}$

for (int $k=0$; $k$ < users.length; $k++$ )

\}

ta.append(">>> "+users[k]+"'n"); 
public void chatNotify(Message $\mathrm{m}$ )

throws RemoteException

\{

if (m.getText() != null)

ta.append(m.getSender() + ": " + m.getText()+"(n");

if (m.getLine() != null \&\& !m.getSender().equals(name))

\} dp.addLine(m.getLine ()$)$;

public static void main(String [] args)

throws RemoteException

\{

ChatClientImpl $c c=$ new ChatClientImpl();

System.out.printIn("Created ChatClientImpl");

cc.registerChatter(); // reg. client with server

cc.setSize (700, 400);

cc.setVisible(true);

\}

\}

$/ /$

import java.util.*; // ServerTalker.java import java.rmi.*;

class ServerTalker extends Thread \{

private List $<$ Message $>$ messages $=$

private ChatServer cs;

new LinkedList<Message $>()$;

public ServerTalker(ChatServer c, String name)

\{

$\mathrm{cs}=\mathrm{c}$;

messages.add(new Message(">>> ",

name + " just connected")); 


\footnotetext{
start();

\}
}

synchronized boolean addMessage(Message e) \{

if ( $\mathrm{cs}==$ null)

\{ System.out.println("Server reference is null"); return false;

\}

messages.add(e);

notifyAll();

return true;

\}

public synchronized void run()

\{

boolean running $=$ true

while (running)

\{

try

$\{$ if (messages.isEmpty()) wait(); cs.postMessage(messages.get(0)); messages.remove(0);

\}

catch (InterruptedException e)

\{ System.out.printIn("Interrupted: " + e); \}

catch (RemoteException e)

\{ System.out.println("Server down?" + e); cS = null;

running $=$ false;

\}

yield(); 
$/ /$

public interface LineSender // LineSender.java

\{

\}

void sendLine(Line In);

/l

import java.awt.*; // DrawPad.java

import java.awt.event. *;

import java.util.*;

import javax.swing. *;

class DrawPad extends JPanel

implements MouseListener, MouseMotionListener

\{

private int lastx, lasty;

private int lastlndex $=0 ; \quad / /$ used to mark drawing list

private java.util.List $<$ Line $>$ lines $=$

new ArrayList<Line $>(500)$;

private LineSender Is;

// client created DrawPad

private Image img;

// for double buffering

private Graphics db;

// to access offscreen buffer

DrawPad(LineSender s)

\{

Is = s;

addMouseListener(this);

addMouseMotionListener(this);

\} 
public void paintComponent(Graphics g)

\{

super.paintComponent(g)

if (img != null)

\section{else}

g.drawlmage(img, 0, 0, this);

$\{\quad$ g.drawString("Initializing...", 5, 30);

render();

\}

void render()

\{

if (img $==$ null)

$\{$ img $=$ this.createlmage $(400,400)$;

$\mathrm{db}=$ img.getGraphics();

db.setColor(Color.white);

db.fillRect $(0,0,400,400)$;

db.setColor(Color.black);

db.drawRect(1, 1, 398, 398); db.drawRect(0, 0, 400, 400);

\}

for (Line In : lines)

db.drawLine(In.x1, In.y1, In.x2, In.y2);

lastIndex = lines.size();

repaint();

\}

void clearScreen()

\{

img = null;

lines.clear();

lastIndex=0;

render();

\} 


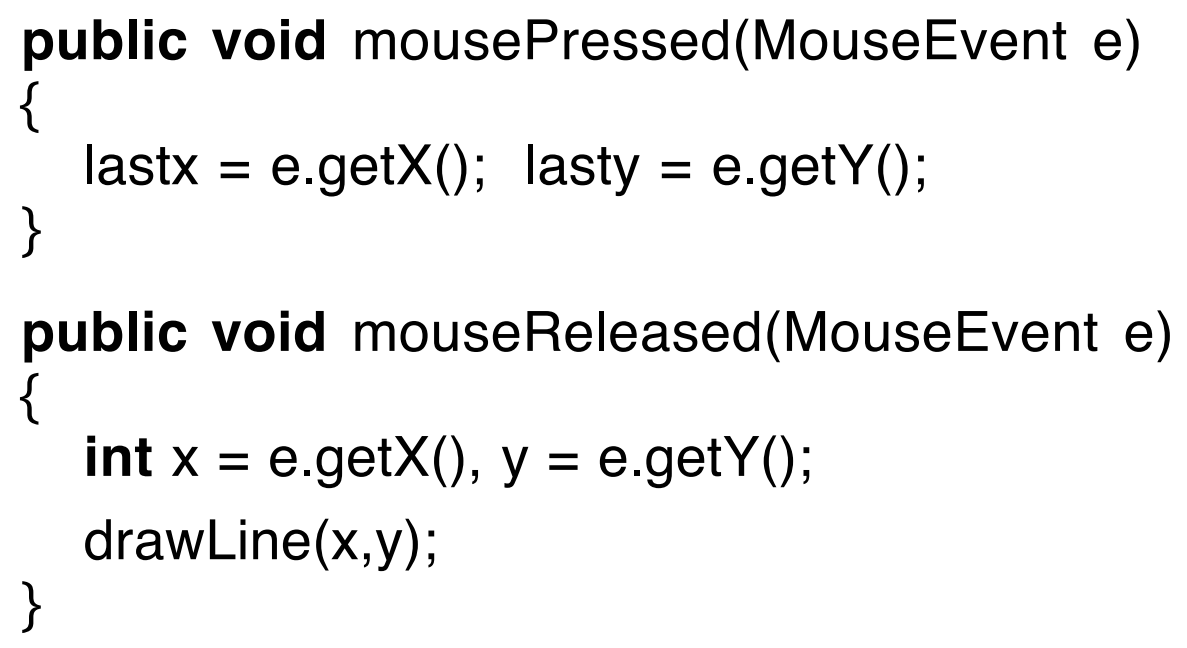

public void mouseMoved(MouseEvent e) \{\}

void drawLine(int $\mathrm{x}$, int $\mathrm{y}$ )

\{

if $(($ Math.abs $($ last $x-x)>0)$ II (Math.abs (lasty-y) $>0))$

\{

Line $\ln =$ new Line $(x, y$, last $x$, lasty);

Is.sendLine(In);

last $\mathrm{x}=\mathrm{x}$; $\quad$ lasty $=\mathrm{y}$;

lines.add(In);

render();

\}

\}

/I 
import java.awt.*;

// NameDialog.java

import java.awt.event. ;

import javax.swing.*;

class NameDialog extends JDialog implements ActionListener \{

private JTextField $\mathrm{tf}=$ new JTextField(20);

private String value $=$ null;

NameDialog(JFrame f, String s, boolean modal)

\{

super(f, s, modal);

Container $\mathrm{cp}=$ getContentPane();

cp.setLayout(new FlowLayout());

add(new JLabel("Enter your name:"));

cp.add(tf);

tf.addActionListener(this);

JButton ok = new JButton("OK");

cp.add(ok);

ok.addActionListener(this);

pack(); setVisible(true);

tf.requestFocus();

\}

public void actionPerformed(ActionEvent e)

\{

if (tf.getText().length() >= 1)

value $=$ tf.getText().trim();

\}

public String getName()

\{

while (value==null II value.equals("'"))

tf.requestFocus();

return value;

\} 
Server Side

ChatServer.java

ChatServerImpl.java

ClientTalker.java

Message.java

Line.java
ChatServer.class

ChatServerlmpl.class

ChatServerImpl_Stub.class

ClientTalker.class

Message.class

Line.class

ChatClient.class

ChatClientImpl_Stub.class

\section{Client Side}

ChatClient.java

ChatClient.class

ChatClientImpl.java

ServerTalker.java

DrawPad.java

LineSender.java

NameDialog.java

ChatClientImpl.class

ChatClientImpl_Stub.class

ServerTalker.class

DrawPad.class

LineSender.class

NameDialog.class

Message.class

Line.class

ChatServer.class

ChatServerlmpl_Stub.class 


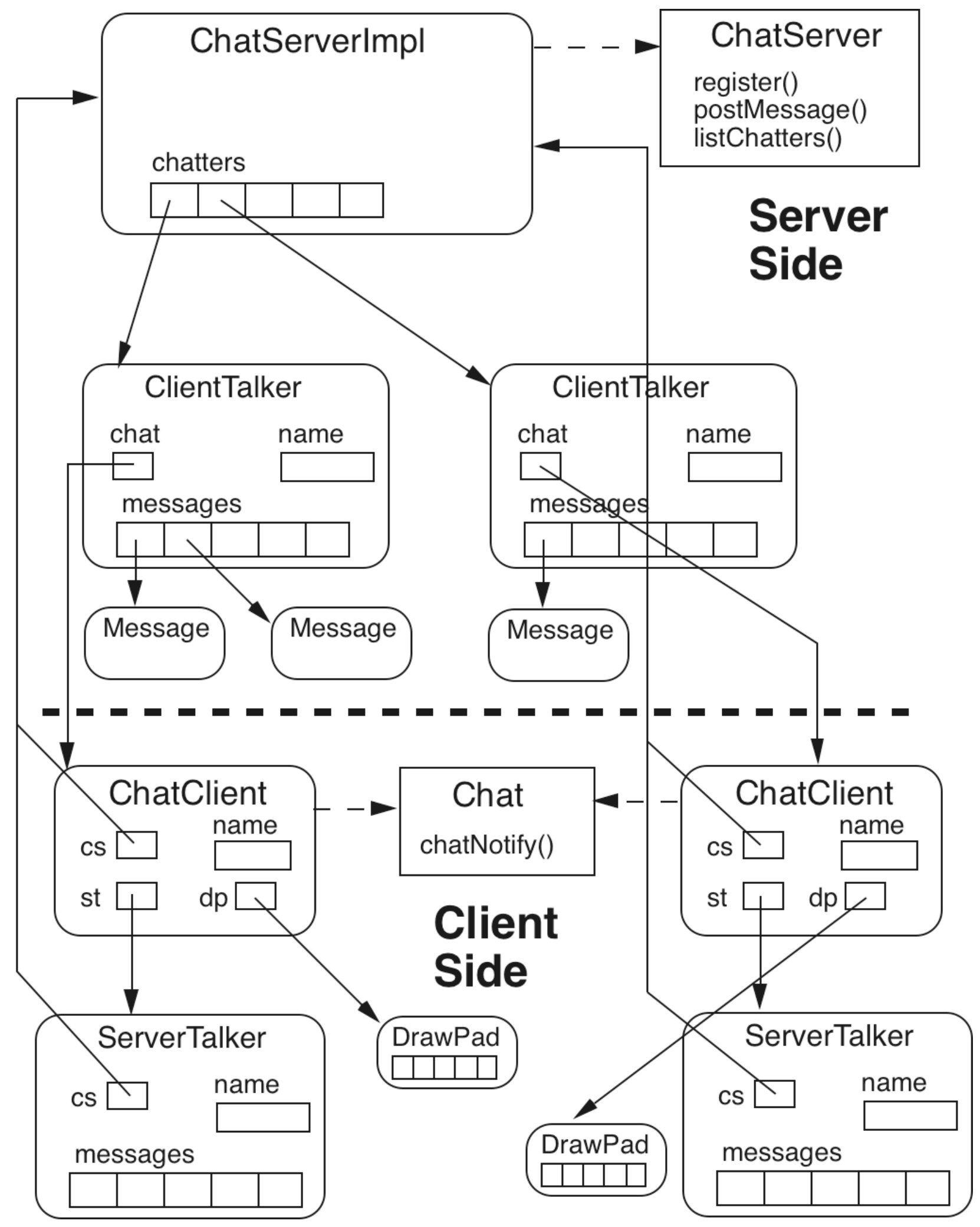




\section{go Files}

$\%$ more server/go1

Jjavac Message.java

ljavac Line.java

echo "Message and Line compiled."

сp Message.class ../client

echo "Message.class copied to client."

cp Line.class ../client

echo "Line.class copied to client."

$\%$ more client/go2

Javac ChatClient.java

Javac LineSender.java

echo "Interfaces ChatClient and LineSender compiled."

cp ChatClient.class ../server

echo "ChatClient.class copied to server."

$\%$ more server/go3

Javac ChatServer.java echo "Interface ChatServer compiled."

сp ChatServer.class ../client

echo "ChatServer.class copied to client."

$\%$ more server/go4

Javac ChatServerImpl.java

ljavac ClientTalker.java echo "ChatServerlmpl and ClientTalker compiled."

rmic -v1.2 ChatServerlmpl echo "Stub file created."

cp ChatServerlmpl_Stub.class ../client echo "Stub copied to client." 


\section{$\%$ more client/go5}

Javac ChatClientImpl.java

ljavac ServerTalker.java

ljavac DrawPad.java

Javac NameDialog.java

echo "ChatClientImpl, ServerTalker, DrawPad,

NameDialog compiled."

rmic -v1.2 ChatClientImpl

echo "Stub file created."

cp ChatClientImpl_Stub.class ../server

echo "Stub copied to server."

$\%$ more server/go6

java ChatServerlmpl\&

echo "Server started."

echo "Do not forget to kill the process when done."

\section{$\%$ more client/go7}

java ChatClientlmpl\&

echo "Client started."

\section{go (in Chat)}

cd server

go1

cd ../client

go2

cd ../server

go3

go4

cd ../client

go5

cd ../server

go6

cd ../client

go7 